\title{
Retinal arterial macroaneurysm: multimodal imaging
}

Sefik Can Ipek ${ }^{l}, M D ;$ Ali Osman Saatci ${ }^{2}, M D$

${ }^{1}$ Department of Ophthalmology, Agri Research and Training Hospital, Agri, Turkey

${ }^{2}$ Department of Ophthalmology, Dokuz Eylul University, Izmir, Turkey

Correspondence and reprint requests:

Prof Ali Osman Saatci, Mustafa Kemal Sahil Bulvarı No:73, A Blok, Daire 9 Narlıdere, Izmir, Turkey.Email: osman.saatci@gmail.com

\section{Abstract}

We present multimodal images of a retinal arterial macroaneurysm in terms of fundus photo, swept source optical coherence tomography, spectral domain optical coherence tomography, fluorescein angiography, and swept source optical coherence tomography angiography.

Key words: Fluorescein angiography; Retinal arterial microaneurysm; Tomography, optical coherence
In March 2020, a 66-year-old woman with a history of stroke, hypertension, and coronary artery disease experienced visual decline in her right eye for 3 weeks. Fundus photo showed a retinal arterial macroaneurysm along the inferotemporal vascular arcade with a cuff of intraretinal and subretinal hemorrhage (Figure a). Swept source optical coherence tomography showed the retinal arterial macroaneurysm extending vertically from the internal limiting membrane to the external limiting membrane, with intraretinal fluid and multiple hyperreflective dots (pearl necklace sign ${ }^{1}$ ) surrounding the inner wall of large cystoid space in the outer plexiform layer (Figure b). Spectral domain optical

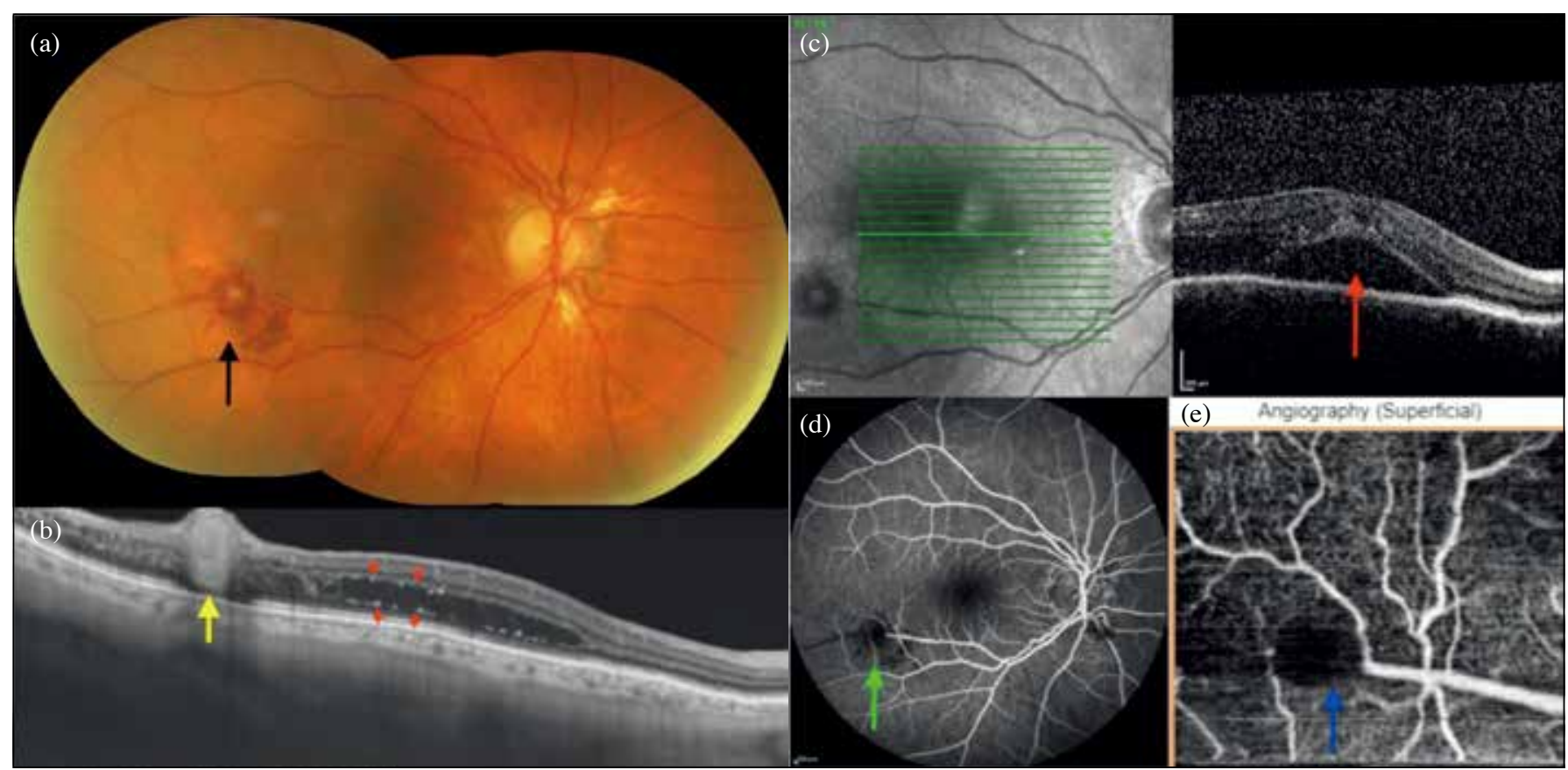

Figure. (a) Fundus photo showing a retinal arterial macroaneurysm (arrow) along the inferotemporal vascular arcade with a cuff of intraretinal and subretinal hemorrhage. (b) Swept source optical coherence tomography showing the retinal arterial macroaneurysm (arrow) extending vertically from the internal limiting membrane to the external limiting membrane, with intraretinal fluid and multiple hyperreflective dots (pearl necklace sign) [arrowheads] surrounding the inner wall of large cystoid space in the outer plexiform layer. (c) Spectral domain optical coherence tomography section through the fovea showing the presence of subretinal fluid (arrow). (d) Fluorescein angiography showing no sign of flow inside the retinal arterial macroaneurysm on the venous phase (arrow). (e) Swept source optical coherence tomography angiography showing sluggish or no flow on the superficial capillary slab (arrow). 
coherence tomography section through the fovea showed the presence of subretinal fluid (Figure c). Fluorescein angiography showed no sign of flow inside the retinal arterial macroaneurysm on the venous phase (Figure d). Swept source optical coherence tomography angiography showed sluggish or no flow on the superficial capillary slab (Figure e). A diagnosis of retinal arteriolar obstruction distal to the retinal arterial macroaneurysm was made. We planned to inject dexamethasone implant for treatment as the patient had a history of stroke, but the patient was lost to followup owing to the Covid-19 pandemic and the subsequent curfew.

\section{Author contributions}

All authors had full access to the data, contributed to the study, approved the final version for publication, and take responsibility for its accuracy and integrity.

\section{Conflict of interest}

The authors have no conflicts of interest to disclose.

\section{Funding/support}

This research received no specific grant from any funding agency in the public, commercial, or not-for-profit sectors."

\section{Patient consent}

The patient was treated in accordance with the Declaration of Helsinki. The patient provided informed consent for the treatment/procedures. The patient provided consent for publication.

\section{Reference}

1. Gelman SK, Freund KB, Shah VP, Sarraf D. The pearl necklace sign: a novel spectral domain optical coherence tomography finding in exudative macular disease. Retina 2014;34:2088-95. Crossref 